\title{
Cost-Efficient Low Latency Communication Infrastructure for Synchrophasor Applications in Smart Grids
}

\author{
Binxu Yang, Konstantinos Vasileios Katsaros, Wei Koong Chai, and George Pavlou
}

\begin{abstract}
With the introduction of distributed renewable energy resources and new loads, such as electric vehicles, the power grid is evolving to become a highly dynamic system that necessitates continuous and fine-grained observability of its operating conditions. In the context of the medium voltage (MV) grid, this has motivated the deployment of phasor measurement units (PMUs), that offer high-precision synchronized grid monitoring, enabling mission-critical applications such as fault detection/location. However, PMU-based applications present stringent delay requirements, raising a significant challenge to the communication infrastructure. In contrast to the high voltage domain, there is no clear vision for the communication and network topologies for the MV grid; a full-fledged optical fiber-based communication infrastructure is a costly approach due to the density of PMUs required. In this study, we focus on the support of low-latency PMU-based applications in the MV domain, identifying and addressing the tradeoff between communication infrastructure deployment costs and the corresponding performance. We study a large set of real MV grid topologies to get an in-depth understanding of the various key latency factors. Building on the gained insights, we propose three algorithms for the careful placement of high capacity links, targeting a balance between deployment costs and achieved latencies. Extensive simulations demonstrate that the proposed algorithms result in low-latency network topologies while reducing deployment costs by up to $80 \%$ in comparison to a ubiquitous deployment of costly high capacity links.
\end{abstract}

Index Terms-Delay, medium voltage power grid, phasor measurement units (PMUs), real topologies, synchronization.

\section{INTRODUCTION}

$\mathbf{T}$ HE energy sector has been undergoing major transformative changes in recent years in order to address pressing concerns in improving energy efficiency of the grid and to reduce overall carbon emissions. The increasing penetration of distributed renewable energy sources (DRER) (e.g., solar/wind farms), the rising deployment of electric vehicles (EV) [1], [2], and active consumer participation into power grid operations (e.g., interactive consumer applications) are pushing today's power grid infrastructure to the limit. The progressive integration of these active components introduces significantly higher system volatility, posing new challenges to system stability, with respect to power quality, voltage regulation, protection [3], and

Manuscript received November 20, 2015; revised March 27, 2016; accepted April 10, 2016. This work supported by the European Commission's Seventh Framework Program FP7-ICT-2011-8 under Grant 318708 (C-DAX) and the CHIST-ERA/EPSRC UK project CONCERT under the Grant EP/L01835/1.

The authors are with the University College London, London WC1E 6EA, U.K. (e-mail: binxu.yang@ucl.ac.uk; k.katsaros@ucl.ac.uk; w.chai@ucl.ac.uk; g.pavlou@ucl.ac.uk).

Digital Object Identifier 10.1109/JSYST.2016.2556420 fault location. In fact, this constitutes a major shift from passive to active distribution networks (ADNs). ${ }^{1}$

To cope with this increasing volatility, distribution network operators (DNOs) aim at the design and development of enhanced cyber-physical systems enabling both the fine-grained monitoring and control of their power grid infrastructure. In the envisioned systems, a communication infrastructure supports the near-real time observability of the power grid conditions, enabling in turn the control of the power grid infrastructure in terms of the aforementioned control operations. In this context, the deployment of high-precision phasor measurement units (PMUs) [4] gains a significant role for DNOs. By supporting high rate, synchronized monitoring of key system parameters, PMUs enable the synchrophasor-based real-time state estimation (RTSE) [5] of the grid, opening the way for fine grained and timely control of the overall system [6]. For example, fault localization enables the instant identification and the subsequent opening/closing of the appropriate breakers, isolating the fault. It has become apparent that the close synergy of communications and the power grid will enable its fine-grained management, supporting the timely adaptation to increasingly dynamic operating conditions.

However, such applications come with stringent end-to-end communication delay requirements, i.e., in the order of a few tens of milliseconds [6]-[8]. In turn, the expected benefits from the envisioned cyber-physical system depend on the ability of the communication infrastructure to actually support these requirements. While high-capacity optical fiber may be typically available on the transmission level (i.e., high voltage (HV) domain), adopting a similar approach on the distribution level (i.e., in the medium voltage (MV) domain) raises significant concerns with respect to the associated costs. Our analysis of a large set of real topologies (cf., Section II-B) shows that the mostly urban environment of the distribution grid calls for a dense deployment of high-capacity communication links, as opposed to the $\mathrm{HV}$ domain [9]. As a result, the full-fledge fiber optic communication deployment in urban environment for MV distribution grid is currently not practical and plagued with various difficulties and prohibitive costs. Recent works have alternatively investigated the use of wireless technologies such as WiMAX and LTE, reporting however concerns about the impact of control plane and medium access control (MAC) layer delays, which is directly

\footnotetext{
${ }^{1} \mathrm{ADNs}$ are defined as distribution networks that have systems in place to control a combination of distributed renewable energy resources like generators, loads and storage. DNOs have the possibility of managing electricity flows via a flexible network topology.
} 
affected by the number of devices accessing the high-capacity wireless channel(s) [8], [10], [11]. On the other hand, the readily available power-line communication (PLC) [12] infrastructure has relatively low costs, but the typically low PLC bandwidth appears as a bottleneck to the timely delivery of delay sensitive monitoring traffic. ${ }^{2}$ Based on the above observations, we identify the tradeoff between the performance gains from the deployment of high bandwidth technologies and the deployment costs (and/or MAC/signaling delay penalties in the case of wireless technologies) associated with wide scale PMU deployment in the MV domain. We highlight that this is the first work in MV domain investigating low latency communication infrastructure for PMU-based applications.

We address this tradeoff by considering the design of a hybrid communication infrastructure, where the existing PLC infrastructure is utilized to reduce the number of high capacity links required to satisfy the low latency requirements along with the associated costs. Our problem resembles a facility location problem, where we seek the minimum number and location of highcapacity links in the MV grid to satisfy our application-level latency constraints. As the problem is known to be NP-hard [13], [14], we turn our attention to heuristic-based solutions. To this end, and in order to guide the design of our solution, we engage in an in-depth analysis of the end-to-end delay ( $\left.T_{\mathrm{e} 2 \mathrm{e}}\right)$ components. Based on a large set of 14 real MV grid topologies operated by a large DNO in the Netherlands, we perform an analysis of important topological characteristics of the MV domain [15], while also paying attention to PMU communication specificities such as the impact of precise PMU data synchronization. Our analysis yields valuable and pragmatic insights for the design of both low-cost and low-latency communication infrastructures for the MV grid, which we embody in the design of three different heuristic-based optimization algorithms. An extensive set of detailed packet level simulations demonstrate the effectiveness of our algorithms.

Our study here is based on our preliminary study in [16]. Summarizing, the contributions of this study are as follows.

1) We identify the tradeoff between communication infrastructure deployment costs and application-level latencies for low-latency PMU-based applications in the MV domain (see Section II). To the best of our knowledge, this is the first study focusing on this tradeoff for the MV domain of the grid.

2) We conduct an in-depth analysis of real MV grid topology characteristics, based on real MV grids comprising 14 primary-substations (P-SS) and 1323 secondarysubstations (S-SS). Our analysis yields specific guidelines for the design of low latency communication infrastructures in the MV domain (see Section III).

3) We identify and analyze the impact of PMU synchronization on the communication latencies (see Section III).

4) We propose three heuristic algorithms for the design of a low-cost and low-latency communication infrastructure in the MV grid (see Section IV). The proposed algorithms are general in nature and applicable to both optical fiber

${ }^{2}$ As also demonstrated by our detailed packet-level simulations in Section II.

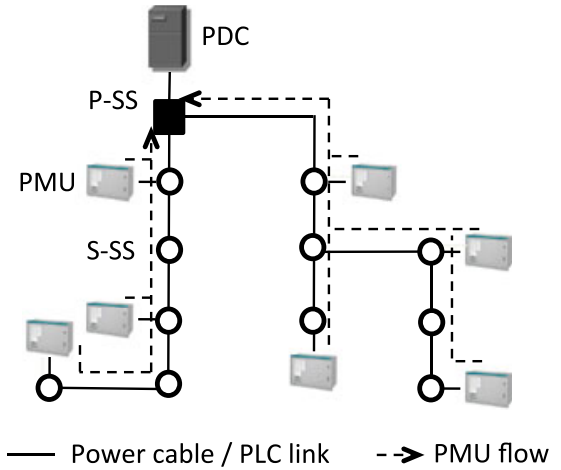

Fig. 1. MV power grid.

and wireless technologies-based communication infrastructures.

5) We demonstrate the effectiveness of our algorithms, i.e., the resulting network topologies satisfy the delay requirements while requiring up to $80 \%$ less high capacity links compared to the HV model of ubiquitous high-capacity link deployment (see Section V).

\section{BACKGROUND AND PROBLEM StATEMENT}

Designing a communication infrastructure for the support of a purpose specific cyber-physical system, such as the smart power grid, necessitates a good understanding of the operational context, in our case of the MV power grid. Fig. 1 provides a high level illustration of a typical MV grid, i.e., the (power) distribution network. A typical MV grid topology has a treelike structure rooted at a P-SS, which is responsible for stepping down the transmission voltage from $\mathrm{HV}$ to MV. Each tree branch emanating from a P-SS corresponds to a distinct feeder (cable) further distributing the MV power to the desired areas through a series of S-SSes, responsible for further stepping down the voltage. The power distribution network consists of multiple such trees rooted at different P-SSes.

\section{A. Delay-Sensitive Synchrophasor Monitoring Applications}

Our study is motivated by the challenge to support three-phase RTSE application. RTSE is considered as an important tool for DNOs as it supports particularly important energy management and protection operations, such as fault detection/localization, postfault management, and voltage control [17], [18]. PMUs enable the support of such applications by monitoring power system parameters (e.g., phase angle, voltage, rate of change of frequency (ROCOF), etc.) at strategically selected S-SSes in the MV $\operatorname{grid}^{3}$ (see Fig 1). All PMUs are GPS-synchronized and stream their measurements to phasor data concentrators (PDCs), which are typically located at the P-SS. PDCs collect, time align, and deliver synchrophasor data to applications such as the RTSE.

\footnotetext{
${ }^{3}$ The selection of PMU locations constitutes a research area on its own (e.g., [19]). Without loss of generality, we consider a scenario with a PMU deployed at approximately every two S-SSes along a feeder (see Fig. 1).
} 
Although typical refresh rates of state estimation processes are of the order of a few minutes, the high system dynamics of ADNs, due to DRERs and EVs, necessitate the fine-grained estimation of system state within a few tens/hundreds of ms [5]. PMU reporting frequencies $(F)$ of 50 or 60 frames/s facilitate this detailed view of the power grid [20]. Based on PMU data semantics [21], a realistic PMU message payload size is 102 B. ${ }^{4}$ Further considering UDP and IP headers, and a 32-B SHA256 message authentication code, the overall data rate for each RTSE PMU flow delivered to the link layer is $64.8 \mathrm{~Kb} / \mathrm{s}$, for $F=50 \mathrm{~Hz}$.

The timely delivery of these measurements is a challenge for the underlying communication infrastructure. In this study, we account for RTSE applications a maximum total latency of $100 \mathrm{~ms}$ [5], [7], [8], including latencies for PMU signal acquisition, PMU synchrophasor estimation and data encapsulation, communication network delay, PDC data frame time alignment, bad data detection, and state estimation [7]. The time budget left for telecommunication network delay $\left(T_{\mathrm{e} 2 \mathrm{e}}\right)$ depends on these latency components and has typically a constraint (denoted as $\left.T_{\max }\right)$ of $20 \mathrm{~ms}$, at a PMU reporting rate of $50 \mathrm{~Hz}$ [5], [8]. It was recently shown that the telecommunication network delay constraint could be further relaxed to $35-55 \mathrm{~ms}$ due to new advancements in state estimation algorithms [7]. Nevertheless, in this study, we focus on $T_{\max }=20 \mathrm{~ms}$, as a more stringent requirement. ${ }^{5}$ At this point, it is important to stress that at the application level, RTSE necessitates the availability of all synchronized PMU measurements within the defined $T_{\max }$. Otherwise, state estimation will suffer in terms of accuracy ; hence, $T_{\max }$ stands for the worst case $T_{\mathrm{e} 2 \mathrm{e}}$ acceptable.

\section{B. Problem Statement}

The support of the identified latency requirements depends heavily on the underlying communication infrastructure, which in turn is largely determined by the locations of the communicating entities and the selected transmission technology. We first consider a baseline communication network model enabled by PLC technologies [12], which, by allowing DNOs to make use of their existing power-line cables as the transmission medium, constitute the most straightforward and low-cost option for the support of communications in the power grid. In this baseline scenario, the communication network topology coincides with the MV power grid topology. We investigate the topological properties of the resulting communication network model based on a set of $14 \mathrm{MV}$ power grid topologies operated by a DNO in the Netherlands. Table I summarizes the basic aggregated topological characteristics of the considered MV grids. Furthermore, in Table II, we present the topological properties per area. Our dataset shows close agreement with literature (e.g., as surveyed in [22]) and thus, representative to general MV grids.

We represent the distribution grid, and the corresponding baseline communication network model, as a set of tree graphs,

\footnotetext{
${ }^{4}$ Considering $\mathrm{PHNMR}=6, \mathrm{ANNMR}=6$ and $\mathrm{DGNMR}=2$, with 32-bit floating-point accuracy [21].

${ }^{5}$ We note though that this is only an input parameter to the proposed algorithms (see Section IV), not affecting their general applicability.
}

TABLE I

SUMMARY OF REAL MV GRID TOPOLOGICAL PROPERTIES OF A LARGE EUROPEAN DNO

\begin{tabular}{lc}
\hline \hline P-SS & 14 \\
S-SS & 1323 \\
Number of edges (cables) & 1426 \\
Average cable length & $498 \mathrm{~m}$ \\
Average node degree & 2.02 \\
\hline \hline
\end{tabular}

TABLE II

Real MV Grid Topological Properties Per AREa

\begin{tabular}{lcccccc}
\hline \hline Grid & $\begin{array}{c}\text { Number of } \\
\text { nodes }\end{array}$ & $\begin{array}{c}\text { Number of } \\
\text { edges }\end{array}$ & Mean node & $\begin{array}{c}\text { Link density } \\
\text { degree }\end{array}$ & $\begin{array}{c}\text { Mean }{ }^{\mathrm{b}} \text { path } \\
\text { length }\end{array}$ & $\begin{array}{c}\text { Mean }^{\mathrm{c}} \\
\text { betweenness }\end{array}$ \\
\hline Area 1 & 187 & 223 & 2.0744 & 0.0128 & 7.3105 & 6.2774 \\
Area 2 & 112 & 134 & 2.7077 & 0.0216 & 7.745 & 6.6869 \\
Area 3 & 36 & 43 & 1.9545 & 0.0683 & 6.2778 & 5.1351 \\
Area 4 & 155 & 177 & 2.1718 & 0.0148 & 7.1290 & 6.0897 \\
Area 5 & 89 & 102 & 2.125 & 0.02604 & 7.1290 & 6.2247 \\
Area 6 & 82 & 82 & 1.9759 & 0.02469 & 3.7195 & 2.6867 \\
Area 7 & 22 & 22 & 1.9130 & 0.0952 & 3.4545 & 2.3478 \\
Area 8 & 177 & 177 & 1.9887 & 0.01136 & 5.3728 & 4.3483 \\
Area 9 & 28 & 28 & 1.9887 & 0.07407 & 5.7857 & 4.6207 \\
Area 10 & 50 & 51 & 2 & 0.04163 & 4.5 & 3.4313 \\
Area 11 & 101 & 101 & 1.9803 & 0.02 & 5.5049 & 4.4608 \\
Area 12 & 98 & 98 & 1.9798 & 0.02061 & 4.55102 & 3.5152 \\
Area 13 & 41 & 41 & 1.9524 & 0.05 & 2.5854 & 1.5476 \\
Area 14 & 145 & 147 & 2.0119 & 0.0141 & 5.2897 & 4.2603 \\
\hline \hline
\end{tabular}

a Path length represents the number of hops from an S-SS to the P-SS.

${ }^{\mathrm{b}}$ Link density $=\frac{|E|}{(|V|-1) * V \mid / 2}$.

${ }^{\mathrm{c} B e t w e e n n e s s ~ r e p r e s e n t s ~ t h e ~ n u m b e r ~ o f ~ s h o r t e s t ~ p a t h s ~ b e t w e e n ~ a n ~ S-S S ~ a n d ~ t h e ~ P-S S ~ t h a t ~}$ involve the measured node.

$G(V, E)$, with $v \in V$ as substations where node $v_{0}$ represents the root (i.e., the P-SS). ${ }^{6}$ The edges, $e \in E$, represent physical cables connecting different SSes. Then, we denote the distance in hop count between $v_{i}$ and $v_{i^{\prime}}$ as $d\left(v_{i}, v_{i^{\prime}}\right)$ with $i \neq i^{\prime}$. Further, let $U$ be the set of nodes (S-SSes) equipped with PMUs, comprising PMU-enabled nodes $v_{i}^{j}$, where $j \in[0 . .|U|-1]$ is the PMU index and $i \in[0 . .|V|-1]$ is the node index. We define $P\left(v_{i}^{j}\right)$ as the shortest path comprising the consecutive edges connecting PMU-enabled node, $v_{i}^{j}$, to $v_{0}$ (see dashed lines in Fig. 1). The length of $P\left(v_{i}^{j}\right)$ is $\left|P\left(v_{i}^{j}\right)\right|=d\left(v_{i}^{j}, v_{0}\right)=d\left(v_{i}, v_{0}\right) .^{7}$

In the PLC-enabled baseline model, PMU flows (dashed arrows in Fig. 1) reach the PDC by traversing their uphill PLC links toward the root of the tree topology. Following the PMU deployment scheme described in Section II-A, for the available MV grid topologies, we simulate the operation of 795 PMUs in a detailed packet-level simulation environment (see Section V). Fig. 2 shows the cumulative fraction of the $T_{\mathrm{e} 2 \mathrm{e}}$ observed at the PDC for a duration of 10 min with PLC bandwidth values: 100 and $500 \mathrm{~Kb} / \mathrm{s}[12] .^{8}$ The vast majority of PMU messages

\footnotetext{
${ }^{6}$ On the communication level, $v$ represent routing/switching devices located at the corresponding S-SSes, forwarding data packets.

${ }^{7}$ For clarity, for the rest of the paper, we simply refer $P\left(v_{i}^{j}\right)$ as $P_{j}$ since there is only one unique path from a PMU to the PDC.

${ }^{8} \mathrm{PLC}$ encompasses a diverse set of technical realizations with different bandwidth values, broadband $500 \mathrm{~Kb} / \mathrm{s}$ being one of them. Our methodology can be applied for different bandwidth values. For extremely low values, lower datarate PMU configurations should obviously be considered.
} 


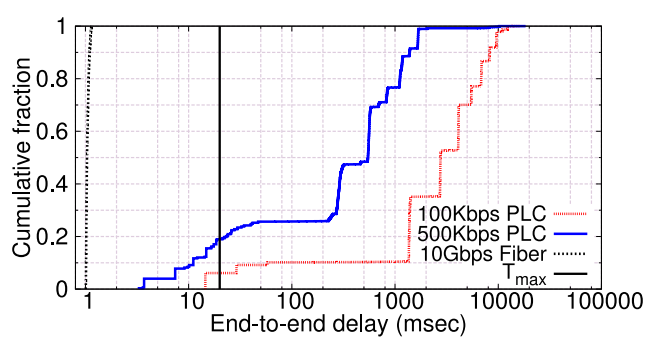

Fig. 2. $T_{\mathrm{e} 2 \mathrm{e}}$ of PMU flows with PLC and optical fiber.

delivered exceeds $T_{\max }$. Clearly, the considered set of applications cannot be supported by PLC technology alone. However, we will show in Section III that limited bandwidth is not the only key delay factor.

We further consider and simulate an optical-fiber based communication network model, following the current practice in HV deployments [9]. In particular, we consider $10 \mathrm{~Gb} / \mathrm{s}$ optical fiber links directly connecting PMU-enabled S-SSes to the PDC at P-SS. As shown in Fig. 2, this communication infrastructure fully conforms to the $T_{\max }$ constraint. However, it necessitates the deployment of 795 optical fiber links in total, representing a significant capital expenditure (CAPEX).

Recent studies have also shown that the adoption of wireless technologies may lead to an increase of medium access delays due to the contention for access to the shared wireless medium, even in cases where no other background traffic is served [8], [10], [11]. This contention and the corresponding delays increase with the number of wireless transmitting devices, i.e., subject to the selected wireless technology, an increased volume of attempts to transmit increases the collision probability, leading to back-off/scheduling delays. Given that existing wireless networks (e.g., cellular (A-)LTE, WiMAX) have been dimensioned for a particular access load, the introduction of additional devices (i.e., PMUs) raises concerns about the aforementioned performance penalties. Of course, increasing frequency reuse with the deployment of smaller cells would reduce contention, for a certain access demand. However, this would obviously come at a significant deployment cost for communication network operators. ${ }^{9}$ The synchronization of PMUs only further exacerbates the contention issue, since it increases collision probabilities and/or limits scheduling flexibility. For all these reasons, it follows that the number of wireless transmitting devices should also be kept to a minimum.

In short, PLC, though readily available, appears unable to support the considered low latency applications, urging for alternative solutions such as the use of modern wireless or highspeed wired technologies. However, the deployment of such technologies incurs a non-negligible capital expenditure and/or performance penalties. In this respect, it becomes apparent that the scale of deployment of high capacity links needs to be carefully considered. Considering this tradeoff between deployment

\footnotetext{
${ }^{9}$ Dedicated, private wireless networks constitute another option for DNOs. However, they are associated with other types of deployment costs, e.g., spectrum license costs. We consider this particular aspect out of the scope of this paper.
}

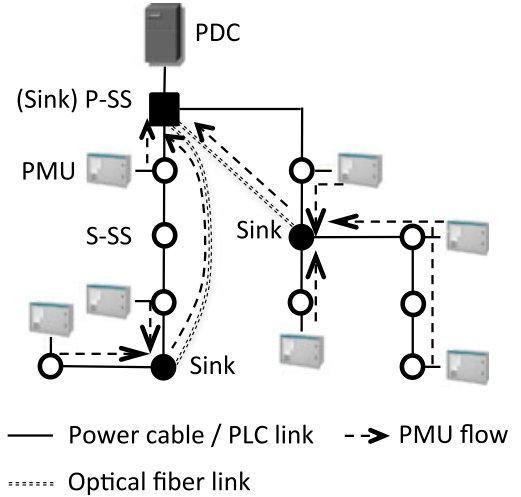

Fig. 3. Hybrid communication infrastructure.

costs and performance, we propose the design of hybrid communication infrastructures that exploit the existing low cost PLC capabilities, while also employing higher bandwidth technologies. The rationale is to take advantage of the availability of PLC to partially accomplish the task of delivering the PMU data flows to the PDC, thus reducing the number of high capacity links in the overall network. Starting from our baseline network model, the objective then becomes to select the minimum sub-set of S-SSes to be equipped with high capacity direct links to the P-SS/PDC (e.g., optical fiber) and act as sink nodes, i.e., aggregating PMU traffic through PLC links. The envisioned scenario is illustrated in Fig. 3.

Let $X_{i}$ with $i \in[0 . .|V|-1]$ be a binary decision variable, set to 1 if node $v_{i}$ is equipped with a high-capacity communication link; we denote such a node with $v_{i}^{k}$ with $k \in[0 . .|S|-1]$, where $S$ be the set of sink nodes. Let also $Y_{\mathrm{jk}}$ with $j \in[0 . .|U|-1]$ and $k \in[0 . .|S|-1]$ be a binary variable set to 1 if a PMU flow from $v_{i}^{j}$ is delivered to a sink node $v_{i^{\prime}}^{k}$. ${ }^{10}$ Then, denoting the end-to-end delay of each PMU flow over path $P_{j}$ (with $j \in[0 . .|U|-1]$ ), as $T_{\mathrm{e} 2 \mathrm{e}}^{j}$, our objective can be loosely expressed as follows:

$$
\begin{array}{rll}
\operatorname{minimize} & \sum_{i} X_{i}, & \\
\text { subject to } & T_{\mathrm{e} 2 \mathrm{e}}^{P_{j}} \leq T_{\max } & \forall j \in[0 . .|U|-1] \\
& \sum_{k} Y_{\mathrm{jk}}=1 & \forall j \in[0 . .|U|-1] \\
& & k \in[0 . .|S|-1] \\
& X_{i} \in\{0,1\} & \forall i \in[0 . .|V|-1] \\
Y_{\mathrm{jk}} \in\{0,1\} & \forall j, k .
\end{array}
$$

The exact nature of the problem and the corresponding solution obviously depend on the first constraint which only roughly expresses the low latency requirement. The second constraint ensures that each PMU-enabled node sends its flow to a single sink node. To assess the hardness of our problem, we can merely express the first constraint by setting an upper limit (i.e., $d_{\max }$ )

\footnotetext{
${ }^{10}$ Note that $i=i^{\prime}$ is allowed i.e., an S-SS can be equipped with both a PMU and a high capacity link.
} 
for the distance between a PMU-enabled node $v_{i}^{j}$ and the corresponding sink location $v_{i^{\prime}}^{k}$. This results in constraint (1) to be re-written as follows (see also Section III-B1):

$$
d\left(v_{i}^{j}, v_{i^{\prime}}^{k}\right) \leq d_{\max } .
$$

Even in this simple case, the resulting problem is a typical NP-hard facility location optimization problem [13], [14] thus turning our attention to heuristic-based solutions. To further explore the problem space and guide the design of our heuristics, we first decompose $T_{\mathrm{e} 2 \mathrm{e}}$ into its constituents and investigate the most important factors impacting them (see Section III). In this effort, we get valuable input from the detailed investigation of our large set of MV topologies. Our analysis yields important insights for the subsequent design of the proposed heuristic algorithms (see Section IV).

\section{ANALYSIS OF IMPACT FACTORS}

Our analysis of the various latency impact factors is enabled by the identification of the various components of $T_{\mathrm{e} 2 \mathrm{e}}$.

1) Processing delay (proc): The time used for operations such as medium adaptation, (de)coding, switching, routing, message authentications codes generation/verification.

2) Propagation delay (prop): Depends on the transmission medium and the distance travelled by the signal. For copper cable, this is typically $5 \mathrm{~ns} / \mathrm{m}$.

3) Transmission delay (trans): The time required to transmit the data and is subject to the bandwidth of the underlying transmission technology.

4) Queuing delay (queue): The time spent by data waiting for transmission at the transmitting devices.

We consider for this analysis a discrete time domain divided into slots with each slot capable of containing exactly one PMU packet. For each delay component $x \in$ \{proc, prop, trans, queue\}, we consider the corresponding per hop delay $t_{x}$. Additionally, we define the aggregate $T_{x}$ of each delay component $x$ over a path $P_{j}$ as $T_{x}=\sum^{\left|P_{j}\right|} t_{x}$.

In the following sections, we investigate the impact of the key factors affecting the aforementioned delay components in order to get insights on where to place high capacity links to achieve the low latency requirement in a cost-efficient manner.

\section{A. Bandwidth}

Bandwidth availability impacts both $t_{\text {trans }} / T_{\text {trans }}$ and $t_{\text {queue }} / T_{\text {queue }}$. Obviously, $t_{\text {trans }} / T_{\text {trans }}$ increase with lower bandwidth values. Moreover, queuing delays perceived at a node increase when the available output bandwidth is lower than the incoming data rate at the node. ${ }^{11}$ Fig. 4(a) shows the cumulative fraction of the $T_{\text {trans }}$ across all PMU-to-PDC paths, for the cases of PLC and optical fiber-based communication infrastructures. For the PLC-based case, $T_{\text {trans }}$ exceeds $T_{\max }$ for $92.91 \%$ and $26.59 \%$ of the transmitted packets for the cases of 100 and $500 \mathrm{~Kb} / \mathrm{s}$, respectively. In the case of optical fiber, we

\footnotetext{
${ }^{11}$ As queuing delays are also related to both topological aspects of the communication network and the synchronization of PMUs, we discuss them in detail in Section III-B
}

see a considerable reduction of accumulated $T_{\text {trans }}$ compared to the PLC case, leaving abundant delay budgets for other delay components. This is a direct consequence of the reduction of $t_{\text {trans }}$ values from 13.52 or $2.70^{\sim} \mathrm{ms}$ for 100 and $500 \mathrm{~Kb} / \mathrm{s}$ PLC datarates, respectively, to only $t_{\text {trans }}=13.52^{\sim} \mu$ s for the case of optical fiber (for the considered payload size and header overheads; see Section II-A).

Our simulations for the baseline PLC scenario (see Section II) also indicate that on average, $T_{\text {queue }}$ accounts for $96.88 \%$ of $T_{\mathrm{e} 2 \mathrm{e}}$, with $T_{\mathrm{trans}}$ and $T_{\text {proc }}$ accounting only for $2.18 \%$ and $0.94 \%$, respectively. ${ }^{12}$ This domination of $T_{\text {queue }}$ on $T_{\mathrm{e} 2 \mathrm{e}}$ implies the lack of sufficient bandwidth to support the PMU traffic. Although the perceived $T_{\text {queue }}$ and $T_{\text {trans }}$ evidently demonstrate the role of the adopted technology's bandwidth, they are still dependent on a series of other factors including the communication network topology and the tight synchronization of PMUs. We further investigate these aspects next.

\section{B. Topology}

1) Path Length: The path length, $\left|P_{j}\right|$, has an important impact on perceived aggregate $T_{x}$ latencies, since lengthy paths accumulate delays on multiple hops. We further use our set of MV topologies to realistically quantify this impact. Fig. 4(b) shows the cumulative fraction of the processing delays accumulated by data packets across all PMU-to-PDC PLC paths (i.e., $\left.P_{j}: \forall j \in[0, \ldots,|U|-1]\right)$, for a range of per node processing delay values, $t_{\text {proc }}$. These values depend on the computational resources of the forwarding devices and can vary significantly, ranging from a few micro-seconds to even milliseconds per packet [23]. If we consider recent overlay approaches [7], [24], these delays may further increase due to the transition of packets from the kernel to the user space. We notice that, subject to $t_{\text {proc }}$, the overall delay penalty $T_{\text {proc }}$ may get close or even exceed $T_{\max }$, even though $T_{\text {proc }}$ constitutes only $0.94 \%$ of $T_{\mathrm{e} 2 \mathrm{e}}$ (for $t_{\text {proc }}=1 \mathrm{~ms}$ ). Similarly, as previously discussed, Fig. 4(a) shows $T_{\text {trans }}$ values close to $T_{\max }$, though $T_{\text {trans }}$ constitutes only $2.18 \%$ of $T_{\text {eze }}$. This is a direct effect of path lengths, which in our topologies have an average and maximum value of 5.84 and 20 hops, respectively.

In essence, these measurements yield an important guideline for the design of low latency communication networks: in the presence of high $t_{\text {proc }}$ values (i.e., in the order of $1 \mathrm{~ms}$ ), bandwidth availability alone may not suffice in keeping $T_{\mathrm{e} 2 \mathrm{e}}$ values low, when paths are considerably long, e.g., interconnecting P/S-SS with optical fiber, following the power grid topology. Moreover, the provisioning of computational resources at each forwarding node should be carefully considered.

Building on these observations, we reformulate the first constraint of our optimization problem (1). Namely, to limit the effect of path lengths on $T_{\mathrm{e} 2 \mathrm{e}}$, we constrain the maximum number of PLC hops by limiting the distance between a PMU and

\footnotetext{
${ }^{12}$ Due to the short distances between S-SSes (see Table I), we omit $t_{\text {prop }} / T_{\text {prop }}$ in the following as it is only in the order of microseconds.
} 


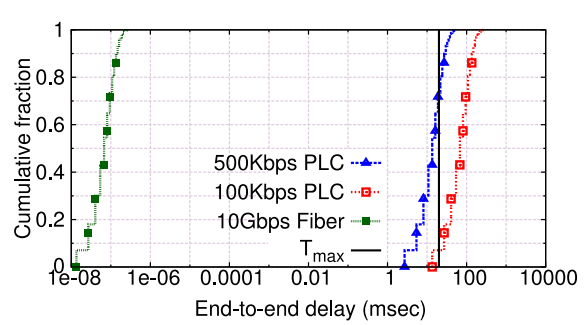

(a)

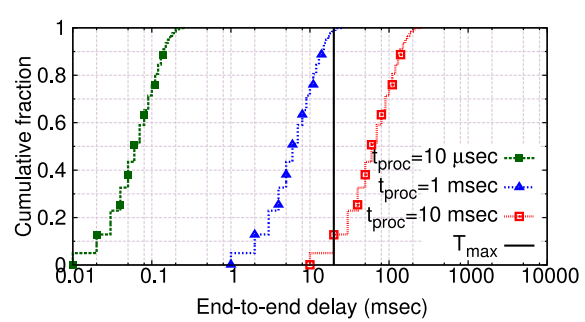

(b)

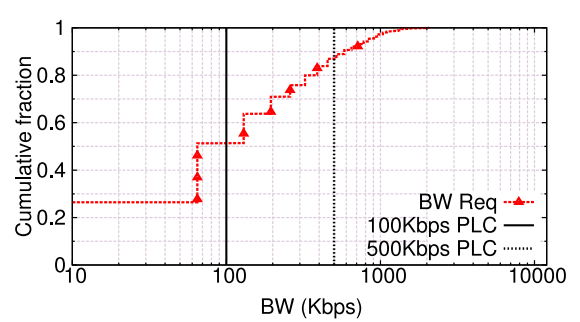

(c)

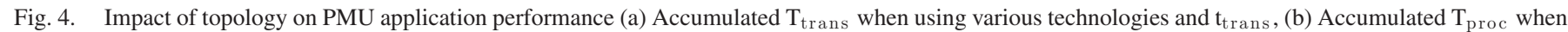
using purely PLC technology with various $t_{\text {proc }}$, (c) PMU bandwidth requirements; traffic volume exceeds PLC capabilities .

its sink node $\left(d_{\max }\right)^{13}$ :

$$
\begin{aligned}
& d\left(v_{i}^{j}, v_{i^{\prime}}^{k}\right) \leq d_{\max }=\left\lfloor\frac{T_{\text {max }}-t_{\text {proc }}}{t_{\text {trans }}+t_{\text {proc }}}\right\rfloor \\
& \forall i \in[0 . .|V|-1] \forall j \in[0 . .|U|-1] \quad \forall k \in[0 . .|S|-1]
\end{aligned}
$$

For the cases of 100 and $500 \mathrm{~Kb} / \mathrm{s}$ PLC, we get $d_{\mathrm{max}}=1$ and 5 hops, respectively, as $d_{\text {max }}$ 's limit values.

2) Application-Level Betweenness: As previously mentioned, $T_{\text {queue }}$ constitutes $96.88 \%$ of $T_{\mathrm{e} 2 \mathrm{e}}$. This delay component depends on the relation between the available and the required bandwidth at each forwarding device. While the former depends on the selected transmission technology, the latter depends on topological aspects of the communication network. Fig. 4(c) shows the cumulative fraction of the total PMU traffic volume aggregated at each PLC link toward the PDC in our MV topologies. Again, we see that a PLC-based infrastructure fails to accommodate the resource requirements as for more than half of the communication nodes, the bandwidth requirements exceed a typical bandwidth value of $100 \mathrm{~Kb} / \mathrm{s}(\approx 10 \%$ for $500 \mathrm{~Kb} / \mathrm{s}$ links).

To better understand this aspect, we introduce the concept of application-level betweenness, $b\left(v_{i}\right)$, as the number of shortest paths $P_{j}$ crossing node, $v_{i}$. Note that $b\left(v_{i}\right)$ is determined both by the topology structure and the placement of the PMUs. In the considered set of MV grid topologies, we observe an average and maximum $b\left(v_{i}\right)$ value of 3.24 and 32 , respectively. Considering a $64.8 \mathrm{~Kb} / \mathrm{s}$ data rate per PMU flow, it is easy to understand the domination of $T_{\text {queue }}$ in $T_{\mathrm{e} 2 \mathrm{e}}$.

Building on this observation, we formulate the next constraint for the design of our hybrid communication network topologies, i.e., we impose an upper bound on application-level betweenness $\left(b_{\max }\right)$ throughout the topology:

$$
b\left(v_{i}\right) \leq b_{\max }=\left\lfloor\frac{\mathrm{BW}}{\mathrm{DR}}\right\rfloor \quad \forall i \in[0 . .|V|-1]
$$

where $\mathrm{BW}$ is the available PLC bandwidth and data rate, $\mathrm{DR}=$ $64.8 \mathrm{~Kb} / \mathrm{s}$. For $\mathrm{BW}=100$ and $500 \mathrm{~Kb} / \mathrm{s}$, this yields $b_{\max }=1$ and 7 , respectively, significantly lower than the observed $b\left(v_{i}\right)$ values in the baseline network model.

\footnotetext{
${ }^{13} t_{\text {prop }}$ (average $\leq 3 \mu$ s in the considered topologies) and $t_{\text {trans }}$ on the sinkto-PDC link ( $\leq 2 \mu \mathrm{s}$ for a $10 \mathrm{~Gb} / \mathrm{s}$ optical fiber link) are considered negligible. However, we account the $t_{\text {proc }}$ for the sink-to-PDC hop.
}

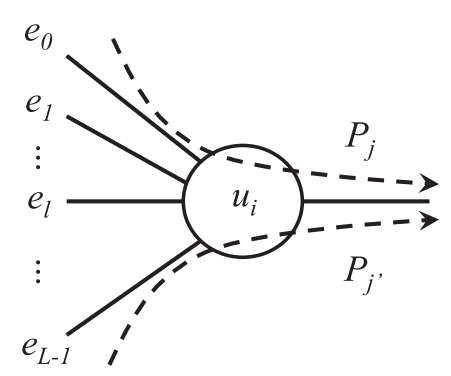

Fig. 5. Example of path $P_{j^{\prime}}$ joining path $P_{j}$ at node $u_{i}$.

\section{PMU Synchronization}

Another factor with significant impact on the $T_{\mathrm{e} 2 \mathrm{e}}$ is the synchronized nature of PMU flows. ${ }^{14}$ As briefly mentioned in Section II, such synchronization may significantly impact the delays for access to the wireless medium. However, PMU synchronization also has an important impact on the baseline PLC network model. Packets originating at different PMUs reach the same forwarding device at (almost) the same time. Consequently, packets wait in the transmission queue for a time linear to $t_{\text {trans }}$, i.e., waiting until all interfering packets from other PMU(s) get transmitted. Our simulation results show that approximately $20 \%$ of PMU flows experience such synchronization problem across $12.45 \%$ of forwarding nodes, inflating the overall observed $T_{\text {queue }}$.

To assess the impact of synchronization, we follow the approach proposed in [25]. Specifically, we focus on the worst-case scenario, i.e., a packet has to wait for all other packets (almost) simultaneously arriving the same node, to get transmitted first. We consider this worst-case scenario as our target is to limit the maximum $T_{\mathrm{e} 2 \mathrm{e}}$ perceived.

We focus on a node of interest, $v$, with $L$ inbound links $e_{l}, l \in[0 . .|L|-1]$ and $e_{f}$ outbound link. Further, a path, $P_{j^{\prime}}$, is said to join path $P_{j}$ when they share the same outgoing edge $e_{f}$ but not an incoming edge $e_{l}$ at the node of interest (see Fig. 5). Let $R_{P_{j}}\left(e_{f}\right)$ be the number of paths, $P_{j^{\prime}}$, that join $P_{j}: \quad \forall j^{\prime} \neq j ; j^{\prime}, j \in[0 . .|U|-1]$, at edge $e_{f}$. Then, the route interference number (RIN) of path $P_{j}$ is defined as follows:

$$
R\left(P_{j}\right)=\sum_{e_{f} \in P_{j}} R_{P_{j}}\left(e_{f}\right)
$$

\footnotetext{
${ }^{14}$ It is worth noting that the synchronization issue did not draw much attention in the HV domain because of the low PMU deployment density and the high bandwidth of the adopted transmission technologies [9].
} 
By counting the number of interfering paths at each forwarding node toward the PDC, RIN allows us to derive the maximum number of times a PMU packet can be delayed due to synchronization in the case where all PMUs send a single packet. In this case, [25] showed that the overall end-to-end $T_{\text {queue }}$ of the packet sent on $P_{j}$ is bounded by $R\left(P_{j}\right)$.

When the $b_{\max }$ constraint is met, the aforementioned single packet case can be generalized into a multipacket case where PMUs send one packet at each measurement interval, $1 / F$. This generalization is possible because measurements taken at one interval will only arrive after all measurement packets from preceding intervals have been transmitted. Furthermore, when the number of flows of each link $e_{f}$ is lower than the maximum $b\left(v_{i}\right): \quad \forall i \in[0 . .|V|-1]$, the worst-case queueing delay of path $P_{j}, T_{\text {queue }}^{P_{j}}$, is bounded by a tighter upper bound compared to RIN [26], [27]. To state this delay bound, let $\beta\left(e_{f}\right)$ denote the number of interfering packets at edge, $e_{f}$ and $Q_{l}$ denote the number of paths from inbound edge $e_{l}$. Then, we express $\beta\left(e_{f}\right)$ and the corresponding worst-case queueing delay, $T_{\text {queue, }}^{P_{j}}$ as follows:

$$
\begin{aligned}
\beta\left(e_{f}\right) & =\sum_{l} Q_{l}-\max _{l}\left\{Q_{l}\right\} \\
T_{\text {queue }}^{P_{j}} & =\sum_{e_{f} \in P_{j}} \beta\left(e_{f}\right) t_{\text {trans }}
\end{aligned}
$$

where function $\max \left\{Q_{l}\right\}$ selects at the outbound edge, $e_{f}$, the maximum number of $Q_{l}$ from all inbound, $e_{l}$.

We can then extend the notion of worst-case queueing delay bound to include $t_{\text {proc }}$ and $t_{\text {trans }}$ along the path to the PDC. Then, $T_{\mathrm{e} 2 \mathrm{e}}^{P_{j}}$ is calculated as follows:

$$
T_{\mathrm{e} 2 \mathrm{e}}^{P_{j}}=\Sigma_{e_{f} \in P_{j}}\left\{\beta\left(e_{f}\right) t_{\mathrm{trans}}+t_{\mathrm{trans}}+t_{\mathrm{proc}}\right\} .
$$

Based on this formulation, we take into account synchronization when satisfying the constraint

$$
T_{\mathrm{e} 2 \mathrm{e}}^{P_{j}}<T_{\max }
$$

\section{Algorithm Design}

Building on the identified constraints, we next describe three heuristic-based algorithms for the design of low latency and low cost hybrid communication infrastructures. Each algorithm is tailored for specific application environments.

1) The path length constraint (PLeC) algorithm selects sink node locations by constraining the length of data delivery paths [with $\left.d_{\max },(5)\right]$, so that the accumulated $T_{\text {proc }}, T_{\text {prop }}$, and $T_{\text {trans }}$ are also capped (see Section III). Since it does not cater for bandwidth availability, this algorithm is most suitable for low DR applications (e.g., low DR PMU reporting) and can be employed for environments where multiple (low DR) applications share the same communication infrastructure.

2) The application-level betweenness and PLeC (AB-PLeC) algorithm selects the sink locations by constraining both path lengths and the number of PMU flows on each PLC link [with $\left.b_{\max },(6)\right]$; therefore, explicitly targeting the reduction of $T_{\text {queue }}$. By adjusting the $b_{\max }$ constraint according to the residual bandwidth of each link, AB-PLeC can be easily adapted to cater for background traffic, i.e., from applications expected to share the same communication infrastructure (e.g., intelligent electronic devicebased monitoring).

3) The flow interference and bandwidth constraint (FIB) algorithm selects the sink locations by explicitly seeking the nodes at which a PMU packet exceeds $T_{\max }$ in the worstcase scenario [see (10)], limiting both $b_{\max }$ and $\beta\left(e_{f}\right)$ values. In contrast to the first two algorithms, FIB takes synchronization into account; however, it is tailored for cases of dedicated communication infrastructure, i.e., no background traffic.

\section{A. PLeC Algorithm}

For the PLeC algorithm, we follow the distance constraint formulation of the $p$-center facility location problem [14]. We define $S=s_{1}, \ldots, s_{m}$ as the set of sink nodes, with $1 \leq m \leq$ $|V|$. Further, let $D\left(S, v_{i}\right)=\min \left\{d\left(s, v_{i}\right): s \in S\right\}$, the distance between each node $v_{i}$ and its nearest sink node. Our objective is to find the minimum set $S$ such that for all $D\left(S, v_{i}\right) \leq d_{\max }$. We solve this problem via the sequential location procedure proposed in [14]. Our algorithm (see Algorithm 1) takes as input the tree topology, $G$ and the distance constraint, $d_{\max }$, and outputs the set of selected sink nodes, $S$, along with set $M$ (see next). For all nodes $v_{i}$, we define a distance value $a_{i}, i \in$ $[0 . .|V|-1]$ and a set $M_{i}$, which contains the nodes that can use node $v_{i}$ as their sink node, under the $d_{\max }$ constraint. We further set $M=\bigcup_{i \in[0 . .|V|-1]} M_{i}$.

The algorithm starts by randomly selecting a leaf node, $v_{l}$ from $G$, along with its parent node $v_{p}$. Traversing the tree hierarchy toward its root, the algorithm updates the distance value $a_{p}$ of nodes $v_{p}$ as in line 9 , until it reaches 0 . Note that the hierarchy is traversed by removing the visited leaf nodes from the topology. When $a_{p}=0$, node $v_{p}$ is added to the sink node set (function $\operatorname{AdD} \operatorname{Sink}\left(G, S, v_{m}\right)$, line 20). In this step, all nodes $v_{i}$ whose minimum hop distance to the new sink $v_{m}$ is below their $a_{i}$ value are added to the $M_{m}$ set. All nodes assigned to the new sink are also removed from the tree..$^{15}$

The outcome of the algorithm consists of the sets $M_{i}$ for each selected sink node $v_{i}$. These sets may overlap with each other in cases where more than one sink nodes reside within the $d_{\text {max }}$ range of some node. At the same time, subject to the exact topological characteristics of tree $G$, sets $M_{i}$ may not all have the same size. This means that a careless assignment of nodes to sinks may result in the overloading of some sink nodes both with respect to their processing and bandwidth capabilities. We address this through a simple node assignment procedure which balances the load between sink nodes. Based on the available $M$ sets, the procedure first produces sets $L_{i}$ which hold the set of all sink nodes within $d_{\max }$ range of each node $v_{i}$. The members

\footnotetext{
${ }^{15}$ This process may result in a forest. Structure $G$ is used for all trees, and getRandomLea fNode ( ) (line 6) returns a leaf node randomly selected from any of the trees.
} 


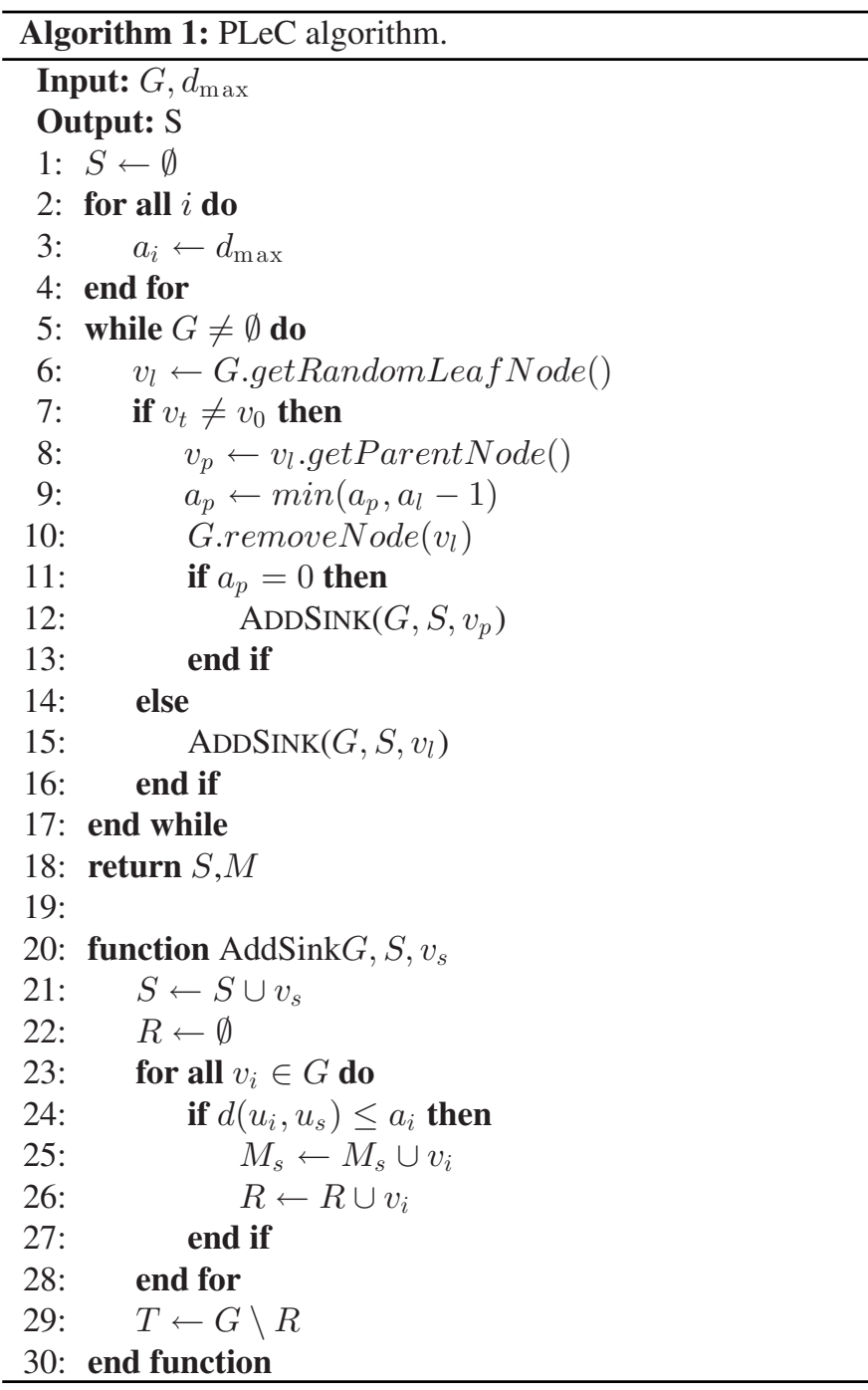

of each $L_{i}$ set are ordered in increasing hop distance to $v_{i}$. The sink node at the smallest distance is selected. When multiple sink nodes are located at the same distance, the algorithm selects the preferred sink node $v_{m}$ with the minimum $M_{m}$ size so as to not overload other sinks which can possibly serve more nodes.

\section{B. AB-PLeC Algorithm}

The AB-PLeC algorithm finds the set of sink locations that constrains the number of PMU flows being forwarded by each PLC link while maintaining the $d_{\max }$ constraint. It takes as input the tree graph topology $G, d_{\max }, b_{\max }$ and the maximum number of PMU flows that can be accommodated by a high bandwidth link connecting a sink node to the PDC, $b_{\max }^{\prime} . b_{\max }^{\prime}$ is set in a similar way to $b_{\max }$, considering the available high-capacity link bandwidth value, and it is, therefore, normally expected to be considerably higher than $b_{\max }$. In addition to $a_{i}$, for each node $v_{i}$, we define $b_{i}$ as the current $b\left(v_{i}\right)$. All $b_{i}$ values are initialized to 0 , unless a PMU is attached to the corresponding node (line 4). The tree topology is traversed from the leafs toward the root node, allowing the forwarding of PMU flows over PLC links up to the point where the uplink capacity of a visited node is exceeded (line 22). This node is then selected to act as a sink location (line 23). PMU flows from additional descendants in the tree may be added, subject to the $b_{\max }^{\prime}$ value (line 15). Visited nodes and sinks are removed from $G$ and the algorithm terminates when all nodes have been removed. Then, each node in the tree can forward its traffic to its closest ancestor sink node.

\section{FIB Algorithm}

Based on the delay bound formulation (9), we propose a heuristic algorithm that constrains $b_{\max }$ and the number of interfering packets of each flow (via $T_{\max }$ ), precisely identifying the required sink locations.

The algorithm takes as input the tree topology $G, b_{\max }, T_{\max }$, $t_{\text {trans }}$, and $t_{\text {proc }}$ and outputs the set $S$ of sink node locations. The algorithm first creates a set $U$ of the nodes equipped with a PMU and computes $b_{i}$ for all $i \in[0 . .|V|-1]$ (line 8). In the second stage, for each PMU-enabled node $v_{i}^{j}$, FIB parses $G$ toward the PDC accumulating the worst-case delay at each node (line 15). When either the calculated delay at a node $v_{m}$ in $P_{j}$ reaches $T_{\max }$ or $b_{\max }$ is violated, the algorithm marks $v_{m}$ as a hotspot.

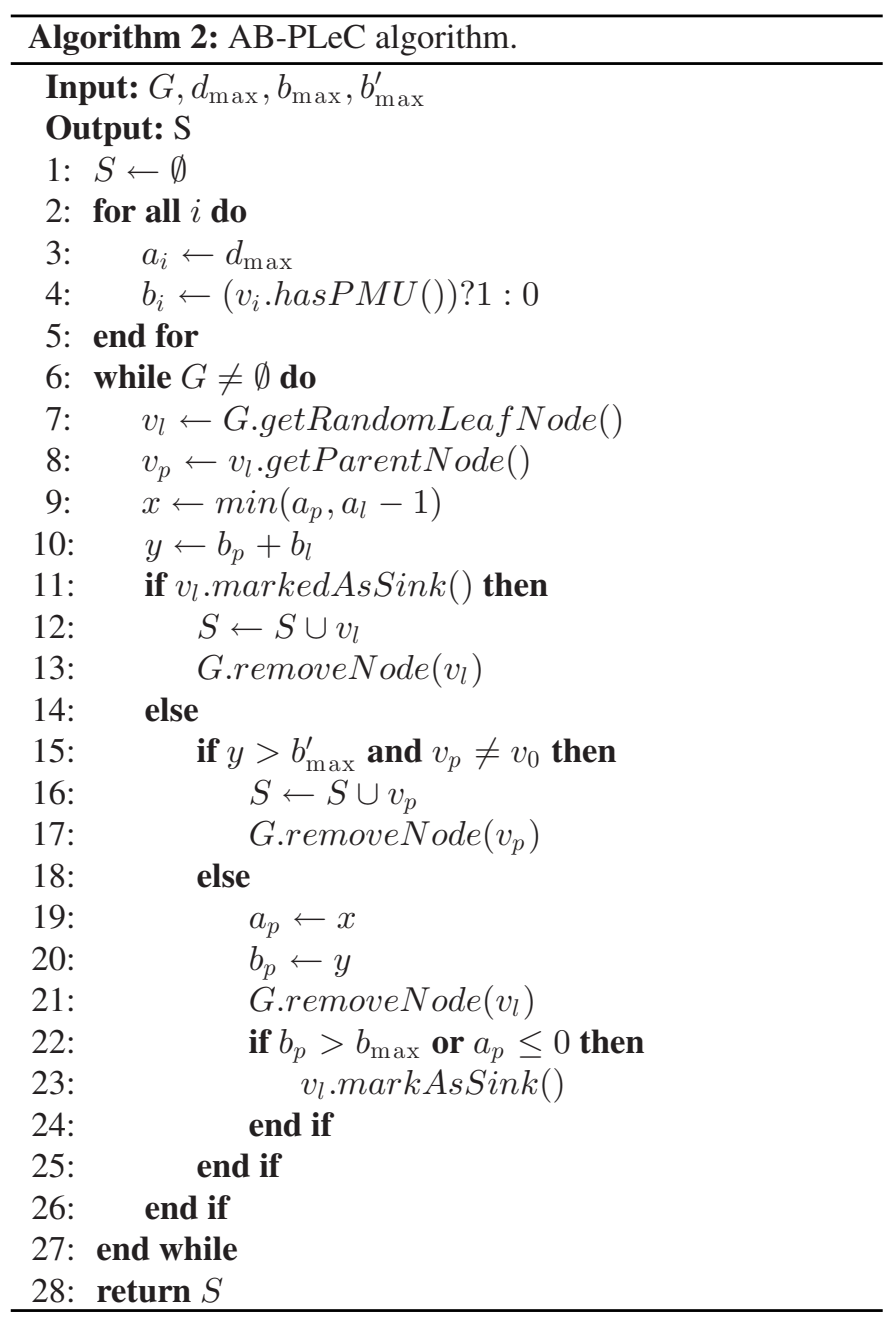

After parsing all nodes in $U$, the FIB algorithm finds the hotspot of each PMU flow. In the third stage, the algorithm selects a 


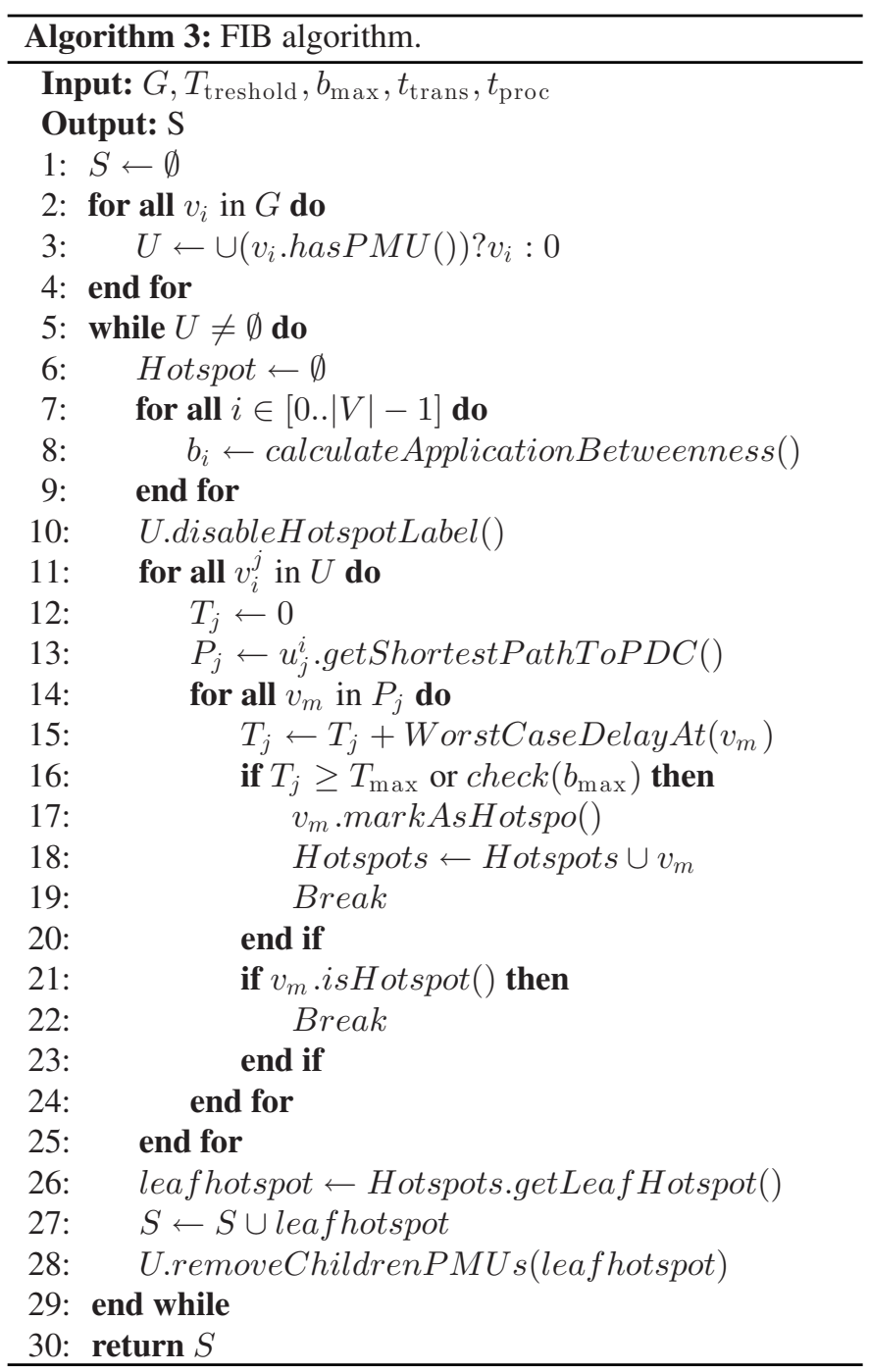

leaf hotspot (i.e., a hotspot with no hotspot descendants) that is farthest to $v_{0}$ and adds it into the sink set, $S$. In the fourth stage, FIB removes the sub-tree rooted at the selected sink location from $G$. The above four stages are repeated until all PMUenabled nodes have been removed from $U$.

\section{Performance Evaluation}

We apply the proposed algorithms on the available tree-like MV power grid topologies and derive a series of alternative communication network topologies under specific constraints. Based on the derived topologies, we perform an extensive set of detailed packet level simulations. We focus on the case of $500 \mathrm{~Kb} / \mathrm{s}$ but similar conclusions apply for the case of $100 \mathrm{~Kb} / \mathrm{s}$. We consider each sink node to be connected to the P-SS with a $10 \mathrm{~Gb} / \mathrm{s}$ optical fiber link and set $t_{\text {proc }}=1 \mathrm{~ms}$. Based on the above, we then get $d_{\max }=5, b_{\max }=7$, and $b_{\max }^{\prime}=147$ as the topological metrics that would conform to the desired $T_{\mathrm{e} 2 \mathrm{e}}$ requirement. Table III summarizes the results for the various derived topologies. We denote the constraints considered by each algorithm as $\operatorname{PleC}\left(d_{\max }\right)$ and $\mathrm{AB}-\mathrm{PLeC}\left(d_{\max }, b_{\max }\right)$. For each topology, we show the percentage of packets measured
TABLE III

SUMMARY OF RESULTING TOPOLOGIES

\begin{tabular}{lcccc}
\hline \hline Sink deployment & $\%$ packets $>T_{\max }$ & $\max T_{\mathrm{e} 2 \mathrm{e}}(\mathrm{ms})$ & \# sink nodes & $\%$ gain \\
\hline PleC(2) & $0 \%$ & 14.8 & 309 & $61.13 \%$ \\
PleC(3) & $0.25 \%$ & 22 & 236 & $70.31 \%$ \\
PleC(4) & $2.16 \%$ & 25.6 & 188 & $76.35 \%$ \\
PleC(5) & $15.94 \%$ & 290 & 160 & $79.87 \%$ \\
AB-PLeC $(3,7)$ & $0 \%$ & 17.5 & 256 & $67.79 \%$ \\
AB-PLeC $(4,7)$ & $0.67 \%$ & 22.9 & 194 & $75.59 \%$ \\
AB-PLeC $(5,7)$ & $20.51 \%$ & 39.1 & 147 & $81.5 \%$ \\
FIB & $0 \%$ & 19.56 & 163 & $79.49 \%$ \\
Full optical fiber & $0 \%$ & 1.14 & 795 & $0 \%$ \\
\hline \hline
\end{tabular}

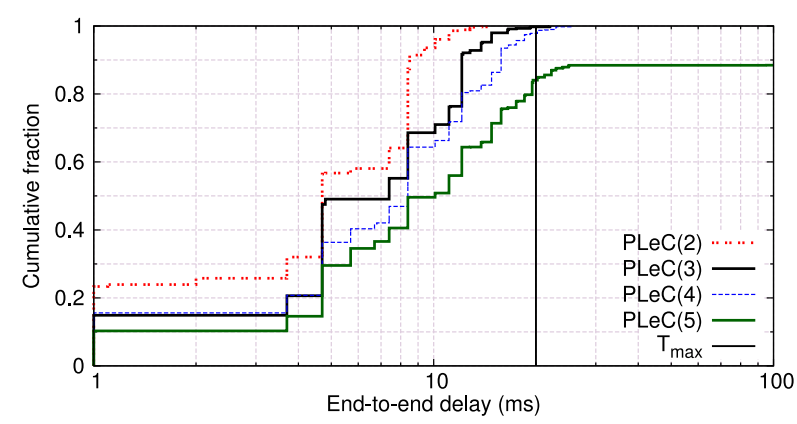

Fig. 6. $\mathrm{CDF}$ of $T_{\mathrm{e} 2 \mathrm{e}}$ for $500 \mathrm{~Kb} / \mathrm{s}$ PLC links: PLeC algorithm.

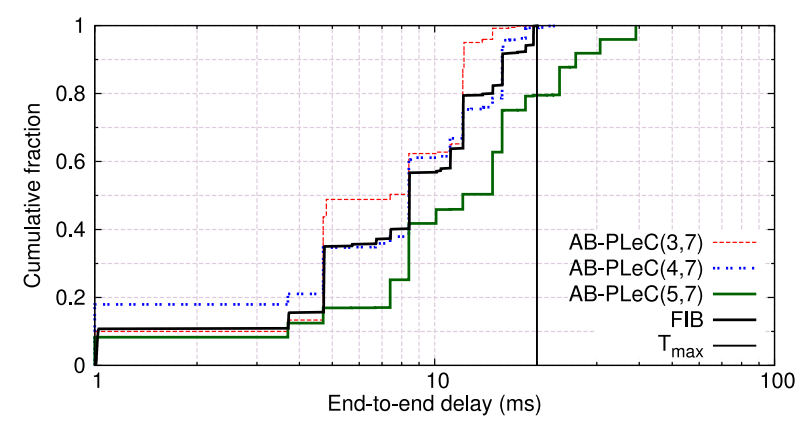

Fig. 7. $\mathrm{CDF}$ of $T_{\mathrm{e} 2 \mathrm{e}}$ for $500 \mathrm{~Kb} / \mathrm{s}$ PLC links: AB-PLeC and FIB algorithm.

to exceed $T_{\max }$, the maximum $T_{\mathrm{e} 2 \mathrm{e}}$, the total number of sink node locations, i.e., the number of high capacity links required, and the gain in terms of the reduction percentage of fiber links compared to the full optical fiber scheme. Figs. 6 and 7 further show the cumulative fraction of $T_{\mathrm{e} 2 \mathrm{e}}$ of all packets, for the various topologies.

We see that FIB, PleC (2), and AB-PLeC $(3,7)$ fully satisfy the delay constraint while requiring only 163, 309, and 256 sink nodes, respectively. This constitutes a reduction in the order of up to $80 \%$ compared to the case of ubiquitous optical fiber deployment, requiring 795 such links. PLeC(2) achieves an overall better performance with median and maximum delay values of 4.7 and $14.8 \mathrm{~ms}$, against 7.4 and $20 \mathrm{~ms}$ of $\mathrm{AB}-\mathrm{PLeC}(3,7)$ respectively. AB-PLeC $(4,7)$ and PLeC(3) closely follow, only slightly exceeding $T_{\max }$ for $<1 \%$ of the measured packets, i.e., by 2.9 and $2 \mathrm{~ms}$, respectively. Also, we see that PLeC(4) and AB$\operatorname{PLeC}(5,7)$ achieve a maximum delay value of 25.6 and $39.1 \mathrm{~ms}$. As discussed in Section II-A, these latencies could be acceptable in cases of improved delay budgets [7], lowering the number of 


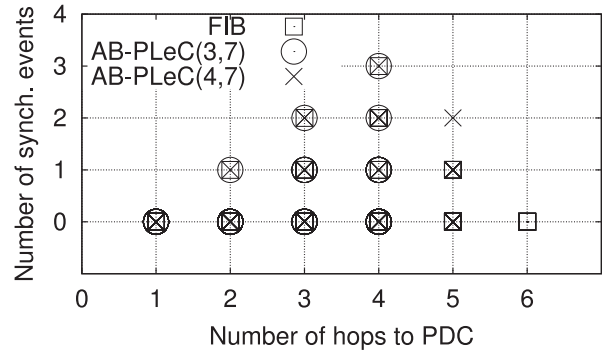

Fig. 8. Each point corresponds to one flow and denotes the length of the path traversed toward the PDC and the number of times the flow may encounter synchronization delays.

sink nodes to 194 and 236, respectively, i.e., an improvement in the other delay components could reduce the high capacity links by approximately $24 \%$ and $23 \%$, respectively.

PLeC(5), PLeC(4), AB-PLeC(4,7), and AB-PLeC(5,7) exceed $T_{\max }$, even though we enforce the $d_{\max }$ and $b_{\max }$ constraint values derived from the considered MV topologies. In the case of PLeC(5), $T_{\mathrm{e} 2 \mathrm{e}}$ reaches a maximum of $290 \mathrm{~ms}$. This is because the PLeC algorithm does not take into account the $b_{\max }$ constraint. Indeed, $b\left(u_{i}\right)$ values (for nonsink nodes) in $\mathrm{PLeC}(5)$ topologies reach a maximum value of 15 , resulting in overloaded uplinks. However, this does not hold for AB-PLeC.

For AB-PLeC $(4,7)$ and $\mathrm{AB}-\mathrm{PLeC}(5,7)$, the nonconformance is attributed to PMU synchronization. Fig. 8 shows for each flow the relation between the length of the corresponding path to the PDC and the number of times the flow may ${ }^{16}$ suffer synchronization events, i.e., its packets arrive at a node (almost) at the same time with packets of other flows. ${ }^{17}$ Topology AB$\operatorname{PLeC}(4,7)$ allows a maximum of four hops to a sink node for all PMU flows (hence five to the PDC), which leads to a delay of 15.82 ms including $d_{\max }\left(t_{\text {trans }}+t_{\text {proc }}\right)$ from PMUs to sinks and a $t_{\text {proc }}+t_{\text {trans }_{10^{-} \mathrm{Gb} / \mathrm{s}}}$ from sinks to the PDC. This leaves $4.184 \mathrm{~ms}$ as the remaining budget for $T_{\max }$. Given this time budget, the maximum number of $t_{\text {trans }}$ a packet could afford to wait in the queue due to synchronization in $\operatorname{AB}-\operatorname{PLeC}(4,7)$ is therefore 1 (i.e., $2.704 \mathrm{~ms}$ ). However, we observe that for $\mathrm{AB}$ PLeC $(4,7)$, some flows may experience synchronization delays twice, thus exceeding $T_{\max }$.

In contrast, the FIB algorithm presents the advantage of explicitly and precisely identifying the locations where $T_{\max }$ is reached. Compared to $\mathrm{AB}-\mathrm{PLeC}(4,7)$, we see that FIB may yield even longer paths than AB-PLeC, however, only for cases of limited synchronization events. For instance, Fig. 8 shows a six-hop path with only one synchronization event. In essence, FIB postpones the selection of a sink location as much as possible, leading to sink nodes closer to the PDC, i.e., utilizing $\mathrm{PLC}$ as much as possible. In contrast, $\mathrm{AB}-\mathrm{PLeC}(3,7)$ constrains the number of hops to sinks to 3 (four hops to PDC), forcing packets that could still use PLC, to use the high capacity links of sink nodes. As a result, a higher number of sink nodes must be unnecessarily deployed, i.e., a $56 \%$ increase of sink nodes against the FIB algorithm.

\footnotetext{
${ }^{16}$ Our analysis in Section III-C focuses on the worst-case scenario, which is experienced by only one of the flows.

${ }^{17}$ Obviously, multiple data points coincide in each case.
}

\section{CONCLUSION AND FUtURE WORK}

The paradigm shift toward ADNs introduces new challenges for the support of smart grid monitoring and control, including low latency communications for mission-critical applications. Though similar challenges have been addressed in the HV domain through the use of high-speed optical fiber links [9], the communication infrastructure landscape in the MV domain remains far from clear. In this paper, based on an extensive set of real MV grid dataset from a large European DNO, we show that the increased density of the MV domain renders the support of low latency applications particularly costly, as opposed to the much sparser HV domain. Proposing the limited use of the readily available PLC technologies, we identify and examine the tradeoff between performance and deployment costs. Drawing on the insights gained from a detailed study of the available topologies, we derive a set of practical guidelines for the design of hybrid, low latency communication infrastructures. Our investigation explicitly identifies, quantifies, and addresses the effect of PMU-device synchronization on queuing delays. Building on our empirical observations, we propose and evaluate three heuristic algorithms that identify the locations in a given grid that should be equipped with high capacity links, striking a balance between low latencies and deployment costs. Enforcing our algorithms on the available MV topologies and additionally engaging in extensive packet-level simulations, we show that the proposed algorithms can indeed satisfy the targeted low latencies while reducing the extend of high capacity link deployment by up to $80 \%$ in comparison to ubiquitous deployment of direct interconnection between PMUs and PDC. We believe our provisioning framework and associated algorithms can help power grid operators in a cost-effective transition toward a smart grid infrastructure.

To the best of our knowledge, this is the first study that identifies, quantifies, and further investigates the tradeoff between performance and deployment costs in the MV domain. Previous work in the area has been centered around the investigation of the topological properties of MV grids [15], [28], or the design of overlay communication networks [7], [24] focused on reliability and reconfiguration issues.

Our future work includes a detailed investigation of additional key performance indicators such as packet loss. To this end, the interplay between transport layer error control mechanisms (such as TCP) and their impact on the achieved latencies and throughput calls for a detailed investigation, in the context of synchrophasor applications. Moreover, the impact of link layer, forward error correction and error control mechanisms (e.g., automatic repeat request) needs to be taken into account in the design of future smart grid communication infrastructures in the MV domain.

\section{ACKNOWLEDGMENT}

The authors alone are responsible for the content of this paper.

\section{REFERENCES}

[1] K. V. Katsaros, W. K. Chai, B. Vieira, and G. Pavlou, "Supporting smart electric vehicle charging with information-centric networking," in Proc. 10th Int. Conf. Heterogeneous Netw. Quality, Rel., Security Robustness, 2014, pp. 174-179. 
[2] Y. Cao, N. Wang, G. Kamel, and Y.-J. Kim, "An electric vehicle charging management scheme based on publish/subscribe communication framework," IEEE Syst. J., DOI: 10.1109/JSYST.2015.2449893.

[3] D. Laverty, D. Morrow, R. Best, and P. Crossley, "Differential ROCOF relay for loss-of-mains protection of renewable generation using phasor measurement over Internet Protocol," in Proc. CIGRE/IEEE PES Joint Symp. Integr. Wide-Scale Renewable Resources Into Power Del. Syst., 2009, pp. 1-7.

[4] P. Myrda and K. Koellner, "NASPInet-The internet for synchrophasors," in Proc. 43rd Hawaii Int. Conf. Syst. Sci., 2010, pp. 1-6.

[5] M. Paolone et al., "A hardware-in-the-loop test platform for the real-time state estimation of active distribution networks using Phasor measurement units," in Proc Cigré SC6 Colloquium, 2014, pp. 1-6.

[6] A. Phadke and J. Thorp, "Communication needs for wide area measurement applications," in Proc. 5th Int. Conf. Critical Infrastructure, 2010, pp. 1-7.

[7] W. K. Chai, "An information-centric communication infrastructure for real-time state estimation of active distribution networks," IEEE Trans. Smart Grid, vol. 6, no. 4, pp. 2134-2146, Jul. 2015.

[8] R. Khan and J. Khan, "Wide area PMU communication over a WiMAX network in the smart grid," in Proc. 3rd Int. Conf. IEEE SmartGridComm, 2012, pp. 187-192.

[9] M. Chenine, K. Zhu, and L. Nordstrom, "Survey on priorities and communication requirements for PMU-based applications in the Nordic Region," in Proc. IEEE Bucharest PowerTech, 2009, pp. 1-8.

[10] P. Cheng, L. Wang, B. Zhen, and S. Wang, "Feasibility study of applying LTE to smart grid," in Proc. IEEE Smart Grid Modeling Simul., 2011, pp. 108-113.

[11] J. Brown and J. Khan, "Performance comparison of LTE FDD and TDD based Smart Grid communications networks for uplink biased traffic," in Proc. IEEE SmartGridComm, 2012, pp. 276-281.

[12] S. Galli, A. Scaglione, and Z. Wang, "Power line communications and the smart grid," in Proc. IEEE SmartGridComm, 2010, pp. 303-308.

[13] M. S. Daskin, "What you should know about location modeling," Naval Res. Logistics, vol. 55, no. 4, pp. 283-294, 2008.

[14] R. L. Francis, T. J. Lowe, and H. D. Ratliff, "Distance constraints for tree network multifacility location problems," Operations Res., vol. 26, no. 4, pp. 570-596, 1978.

[15] G. A. Pagani and M. Aiello, "Towards decentralization: A topological investigation of the medium and low voltage grids," IEEE Trans. Smart Grid, vol. 2, no. 3, pp. 538-547, Sep. 2011.

[16] K. Katsaros, B. Yang, W. K. Chai, and G. Pavlou, "Low latency communication infrastructure for synchrophasor applications in distribution networks," in Proc. IEEE SmartGridComm, 2014, pp. 392-397.

[17] K. Christakou, J. LeBoudec, M. Paolone, and D.-C. Tomozei, "Efficient computation of sensitivity coefficients of node voltages and line currents in unbalanced radial electrical distribution networks," IEEE Trans. Smart Grid, vol. 4, no. 2, pp. 741-750, Jun. 2013.

[18] IEEE Guide for Design, Operation, and Integration of Distributed Resource Island Systems with Electric Power Systems, IEEE Std 1547.42011, pp. 1-54, 2011.

[19] Q. Li et al., "An information-theoretic approach to PMU placement in electric power systems," IEEE Trans. Smart Grid, vol. 4, no. 1, pp. 446456, Mar. 2013

[20] IEEE, IEEE Standard for Synchrophasor Measurements for Power Systems, IEEE Standard C37.118.1, 2011

[21] IEEE, IEEE Standard for Synchrophasor Data Transfer for Power Systems, IEEE Standard C37.118.2, 2011.

[22] G. A. Pagani and M. Aiello, "The power grid as a complex network: a survey," Physica A: Statist. Mech. Appl., vol. 392, no. 11, pp. 2688-2700, 2013.

[23] R. Ramaswamy, N. Weng, and T. Wolf, "Characterizing network processing delay," in Proc. Global Telecommunications Conf., 2004, vol. 3, pp. 1629-1634.

[24] K. V. Katsaros et al., "Information-centric networking for machine-tomachine data delivery-A case study in smart grid applications," IEEE Netw. Mag., vol. 28, no. 3, pp. 58-64, May/Jun. 2014.

[25] I. Chlamtac, A. Farago, H. Zhang, and A. Fumagalli, "A deterministic approach to the end-to-end analysis of packet flows in connection-oriented networks," IEEE/ACM Trans. Netw., vol. 6, no. 4, pp. 422-431, Aug. 1998.

[26] J.-Y. Le Boudec and G. Hebuterne, "Comments on "a deterministic approach to the end-to-end analysis of packet flows in connection oriented networks"," IEEE/ACM Trans. Netw., vol. 8, no. 1, pp. 121-124, Feb. 2000

[27] H. Zhang, "A note on deterministic end-to-end delay analysis in connection oriented networks," in Proc. IEEE Int. Conf. Commun., 1999, vol. 2, pp. 1223-1227.
[28] W. K. Chai, V. Kyritsis, K. Katsaros, and G. Pavlou., "Resilience of interdependent communication and power distribution networks against cascading failures," presented at the 15th IFIP Networking, Vienna, Austria, 2016.

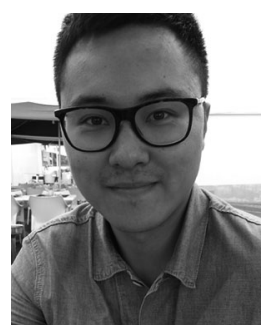

Binxu Yang received the B.S. degree in telecommunications from Xidian University, Xian, China, the French Engineering degree from Telecom Bretagne, Plouzane, France, and the M.Res. degree (Distinction) from University College London (UCL), London, U.K, in 2010, 2013, and 2014, respectively. He is currently working toward the Ph.D. degree from the Department of Electronic and Electrical Engineering, UCL.

His current research interests include smart grid communications, mobile edge computing, and resource allocation in network function virtualization.

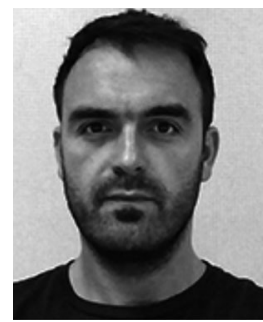

Konstantinos Vasileios Katsaros received the B.Sc. degree in informatics in 2003, the M.Sc. (Hons.) degree in 2005, and the Ph.D. degree in computer science from the Athens University of Economics and Business, Athens, Greece, in 2010.

He has worked in the areas of cloud networking, mobile grid computing, and multicast/broadcast service provision over cellular networks. His research interests include information-centric networking and smart grid communications. He is a currently a Research Associate at the Department of Electronic and Electrical Engineering, University College London, London, U.K.

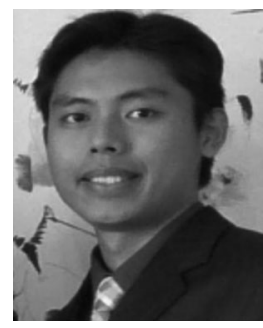

Wei Koong Chai received the B.Eng. degree in electrical engineering from the Universiti Teknologi Malaysia, Johor Bahru, Malaysia, in 2000, and both the M.Sc. (Distinction) and the Ph.D. degrees from the University of Surrey, Surrey, U.K., in 2002 and 2008 , respectively.

He is currently a Senior Research Associate at the Department of Electronic and Electrical Engineering, University College London, London, U.K. His research spans across heterogeneous networks including wired/wireless networks and cyber physical systems such as smart grid. His current research interests include informationcentric networking, smart grid communication, quality of service, resource management (e.g., for satellite networks and wireless mesh networks), crosslayer design (specifically on interaction of protocols at different layers), traffic engineering, and network optimization.

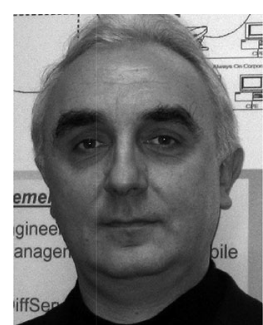

George Pavlou received the Diploma degree in engineering from the National Technical University of Athens, Athens, Greece, and the M.Sc. and Ph.D. degrees in computer science from University College London, London, U.K.

$\mathrm{He}$ is a Professor of communication networks in the Department of Electronic and Electrical Engineering, University College London, where he coordinates research activities in networking and network management. His research interests include networking and network management, including aspects such as traffic engineering, quality of service management, autonomic networking, information-centric networking, grid networking, and software-defined networks. He has been instrumental in a number of European and U.K. research projects that produced significant results with real-world uptake and has contributed to standardization activities in ISO, ITU-T, and IETF. He has been on the editorial board of a number of key journals in these areas, he is the Chief Editor of the bi-annual IEEE Communications network and service management series and in 2011 he received the Daniel Stokesbury Award for distinguished technical contribution to the growth of the network management field. 